\title{
A Unique Method of Plantar Forefoot Ulcer Closure using the Ilizarov Device: Series of 11 Patients with Leprosy
}

\author{
by B. Jagannath Kamath, M.S. (Ortho) ${ }_{1} \square$, Praveen Bhardwaj, M.S., (Ortho) ${ }^{2}$
}

The Foot \& Ankle Journal 1 (1): 3

Original article: Published online 1/1/2008

Introduction: Recurrent plantar ulcer is a common and serious problem of anesthetic feet in leprosy. There are many methods described in the literature to deal this problem, but it still remains a great challenge for the treating surgeon. The problem lies not only in attaining the coverage and healing of the ulcer but also to prevent its recurrence. The skin of the forefoot is a specialized one and hence procedures aimed at providing skin from elsewhere will tend to fail. The local flaps described in the literature are effective, but forms a major surgery for this group of patients, requires an expert to perform and are not devoid of complications, which if occur can be disastrous. We are herein describing a technique of closure of these forefoot ulcers, which makes use of the biomechanical properties of the skin and provided closure of these ulcers by slow and sustained stretching of the surrounding natural skin. It thus provides the specialized plantar skin coverage for the ulcers.

Method: We have used this technique in eleven leprosy patients with forefoot ulcers. Four patients had ulcers under the head of first metatarsal, four under the heads of first and second metatarsals, two under the second metatarsal head and one under the heads of fifth metatarsals. Size of the ulcers at its maximum length ranged from $2-4 \mathrm{~cm}$.

Results: The technique successfully resulted in healing of all the ulcers without any significant complication. All the ulcers could be closed in ten days and all of them healed well within six weeks.

Conclusion: We found the technique to be very effective. It providing the specialized plantar skin for healing the ulcers is most attractive feature. The technique is simple and free of any major complication. The device used to achieve the closure is very inexpensive, can be easily made and is easy to use.

Key Words: Forefoot ulcers in leprosy; technique of closure; stretching; provides specialized skin cover; simple, inexpensive and effective.

\footnotetext{
Address correspondence to: Dr. B. Jagannath Kamath. Jyothi Mansion, Opposite Prabhat Theatre, K. S. Rao Road, Mangalore, India. Pin- 575001. Phone: 91-0824-2440233; Mobile: 91-9845235747

E-mail: bjkamath@satyam.net.in

${ }^{1}$ Associate Professor of Orthopaedics, Kasturba Medical College, Mangalore, Karnataka, India.

${ }^{2}$ Assistant Professor of Orthopaedics, Kasturba Medical College, Mangalore, Karnataka, India.

E-MAIL: drpb12@yahoo.co.in
} 
$\mathbf{R}_{\text {ecurrent plantar ulceration is a frequently seen }}$ problem in anesthetic feet of leprosy. The seriousness of these ulcers lie in the fact that it can be difficult to keep the ulcers healed. Management of these chronic ulcers can be very frustrating for the patient and for those involved in their treatment. Ulcers occur in about 30 percent of the patients suffering with leprosy and are most commonly seen at the forefoot. ${ }^{1}$ The factors contributing to the onset of these ulcers are impaired sensation, atrophy and fibrosis of muscles of foot and alteration of sympathetic enervation that in turn produces dryness, anhidrosis and hyperkeratosis. As the intrinsic muscles of the foot, which are secondary stabilizers of the metarsophalangeal joints become ineffective, it results in clawing of the toes making the heads of the metatarsals very prominent because of hyperextension of the metacarpophalangeal joints and flexion at the interphalangeal joints. Additionally, the loss of transverse and longitudinal arches of the foot increases the irregular distribution of the weight across the midfoot and metatarsal heads. Ulcers are more common on the plantar forefoot because this is where the greatest forces are concentrated. Ulcers are most common under the first metatarsal head; they also less commonly appear under the second and fifth metatarsal heads. The tissues in this area are highly specialized for the purpose of weight bearing and hence are difficult to reconstruct. ${ }^{2}$ Any procedure to cover the defect in this area should ideally provide a stable local tissue. The distant flaps or free flaps described to cover these areas in our view fail because they lack the special architecture for absorbing impact and shear which the plantar skin is subjected to. Quite a number of local flaps are described to get the specialized plantar skin to cover the skin defects. ${ }^{2-4}$
These flaps are quite successful, but are technically demanding as the plantar skin allows very little mobilization and if the flaps fail it leaves one in disastrous situation.

Some skin stretching techniques have been described in recent past to close skin defects. ${ }^{5-10}$ These techniques utilize viscoelastic properties of skin. Biomechanical properties of skin like mechanical creep, recruitment and stress relaxation allows the skin to stretch. We have used this ability of the skin to close the forefoot ulcers in leprosy patients. The technique described is simple, effective and inexpensive. It has given very satisfying results in our experience.

\section{Materials and Methods}

Patient selection is very crucial and may dictate the final results. We recommend the procedure for:

1. Ulcers which are not grossly infected

2. The ulcer should not be extending to the bone

3. Size of the ulcer should not exceed $4 \mathrm{~cm}$

4. No diabetes mellitus

5. No peripheral vascular disease

We had eleven cases, which met the criteria mentioned above. Procedure was performed for all these patients; details are described in the table 1.

Thorough debridement and freshening of the edges is mandatory as the highly keratinized scar tissue accompanies the plantar ulcers, which must be excised. We avoid excessive undermining of skin margins as this could risk their viability when a strong stretching force is employed for obtaining extra skin. Also K-wire assembly will be unstable if excessive undermining of the margins is done and may even make insertion of $\mathrm{K}$-wires technically difficult. 


\begin{tabular}{|c|c|c|c|}
\hline Case No. & Size of the Ulcer & $\begin{array}{l}\text { Site of the Ulcer } \\
\text { (Under the Head of) }\end{array}$ & $\begin{array}{l}\text { Time taken for } \\
\text { closure }\end{array}$ \\
\hline 1. & $2 \mathrm{~cm}$ & First metatarsal & 5 days \\
\hline 2. & $2.5 \mathrm{~cm}$ & First metatarsal & 5 days \\
\hline 3. & $2.5 \mathrm{~cm}$ & Fifth metatarsal & 6 days \\
\hline 4. & $3.5 \mathrm{~cm}$ & First \& second metatarsals & 8 days \\
\hline 5. & $3 \mathrm{~cm}$ & Second metatarsal & 8 days \\
\hline 6. & $4 \mathrm{~cm}$ & $\begin{array}{l}\text { First, Second \& third } \\
\text { metatarsals }\end{array}$ & 10 days \\
\hline 7. & $3.5 \mathrm{~cm}$ & First \& second metatarsals & 9 days \\
\hline 8. & $4 \mathrm{~cm}$ & First metatarsal & 10 days \\
\hline 9. & $2.5 \mathrm{~cm}$ & Second metatarsal & 7 days \\
\hline 10. & $3.5 \mathrm{~cm}$ & First \& second metatarsals & 8 days \\
\hline 11. & $3 \mathrm{~cm}$ & First metatarsal & 8 days \\
\hline
\end{tabular}

Table 1: Patient details and time taken for ulcer closure.

\section{Description of Device}

The Ilizarov device consists of :

1. Two K-wires of size 1.5 to $1.8 \mathrm{~mm}$ (Figure 1 A)

2. Two specially designed custom-made K-wire holding bolts, which are without any threads and are free to slide over the threaded rods. $\mathrm{K}$-wires are transfixed to these bolts by using either inbuilt or external bolts as shown in the figure no.1. The corners of the bolts are rounded to prevent the sharp edges abrading the skin. (Figure $1 \mathrm{~B}$ )

3. Two threaded rods used in Ilizarov's apparatus in Orthopedic surgery (Figure $1 \mathrm{C}$ )

4. Eight threaded bolts used in Ilizarov's apparatus, which can be threaded on to the rod (Figure 1 D)

\section{Technique}

An example of a forefoot ulcer is shown preoperatively. (Figure 2) The K-wires are passed on the either side of the ulcer at a distance of about one $\mathrm{cm}$ from the edge deep enough to engage in the dermis. (Figure 3) It is important to have the K-wires deep enough in the dermis and at equal depth throughout its extent to exert a strong and uniform approximating force. The Kwires are secured onto the $\mathrm{K}$-wire holding bolts. (Figure $1 \& 3$ ) Threaded rods are passed into the $\mathrm{K}$-wire holding bolts on both the sides and are secured in place by two threaded bolts on the outer aspect of each K-wire holding bolt. (Figure 3) These threaded bolts will thus approximate the $\mathrm{K}$-wire holding bolts and hence the $\mathrm{K}$-wires as they are tightened. 


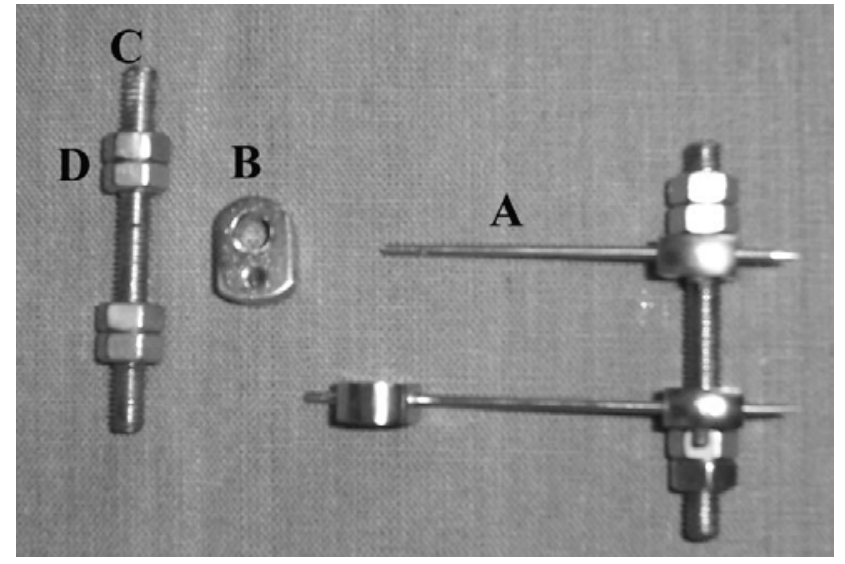

Figure 1 Components of the device: A: K-wires; B: K-wire holding bolt; C: threaded rod; D: nuts.

We start approximating the wound on the first postoperative day. Each single turn of the threaded bolt causes the K-wires on either side to approximate the wound by $1 \mathrm{~mm}$ on both sides and causing $2 \mathrm{~mm}$ decrease in the wound size.

The speed of approximation depends of the skin condition, which is vigilantly monitored. Skin pallor, tautness of skin and shininess and excessive pain are the indicators of temporary stoppage of approximation process. Generally in the beginning we are able to approximate about 5$6 \mathrm{~mm}$ and gradually decrease it as the time passes by. In all the cases we have been able to achieve complete closure in 10 days or less. We have experienced that it is possible to achieve a greater degree of closure in medio-lateral direction than the antero-posterior direction. After approximation is achieved the skin edges are sutured and the fixator is maintained for another week. (Figure 4) The patient is kept non-weight bearing for another two weeks. Patients are then advised regarding foot care and hygiene and are asked to avoid static standing for more than 10 minutes at a time. Prevention of recurrence is very crucial. Secondary procedures like metatarsal osteotomy may be done if required to prevent recurrence once the ulcer is closed. (Figure 5)

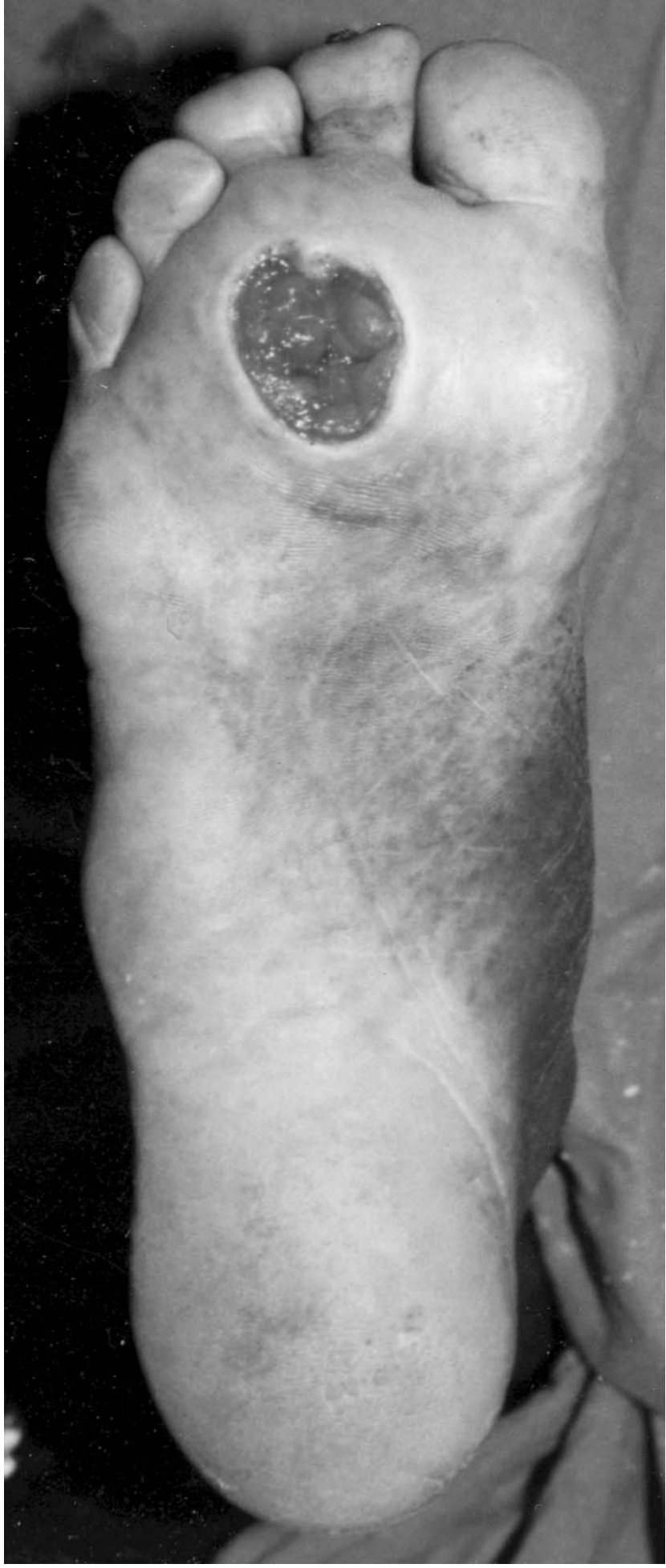

Figure 2 Preoperative photograph of a patient showing the ulcer present under the heads of metatarsals extending from base of first metatarsal to that of the third metatarsal. 


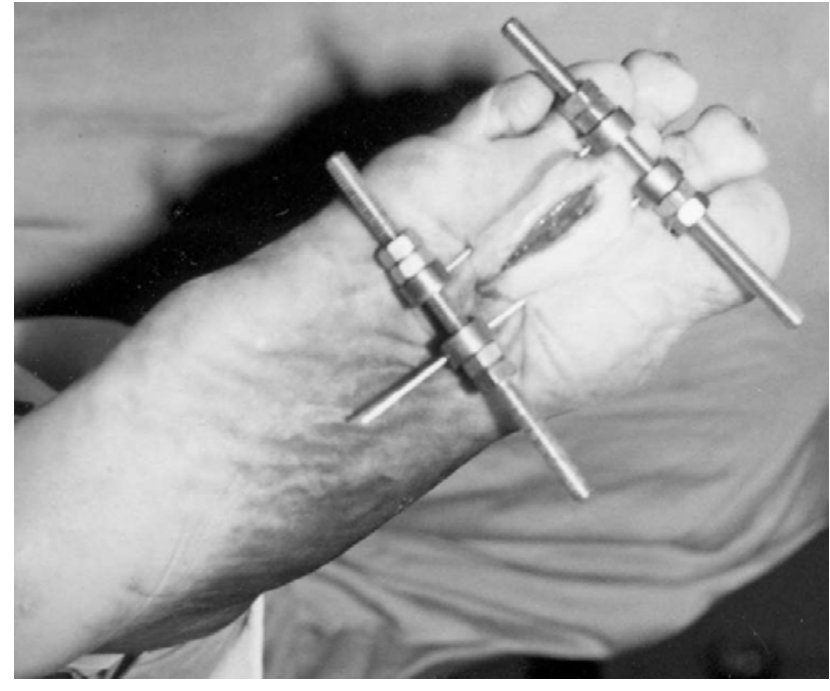

Figure 3 Photograph taken after initial approximation of the wound as was easily possible in the early postoperative days.

\section{Discussion}

Treatment of ulcers in leprosy remains a challenge to the treating surgeon. The various options available are casts, modified foot wears, local flaps and distant flaps.

The non-operative methods are useful only in cases where the size of the ulcer is small and thus for larger ulcers surgery is the only option. The distant flaps fail to provide the specialized plantar skin and hence are likely to fail. The various local flaps described in the literature are quite handy but form a major surgical procedure requiring an expert to perform them and have grave consequence if they fail. The application and principle of gaining tissue by recruitment using force is not new and has been successfully applied to wound coverage problem for years. ${ }^{5-10}$ Skin stretching results in significant histomorphological changes in collagen fibers of the dermis and results in their rapid realigning in response to the stretching force and become aligned in the direction of the stretching force, perpendicular to the wound margin. ${ }^{11}$

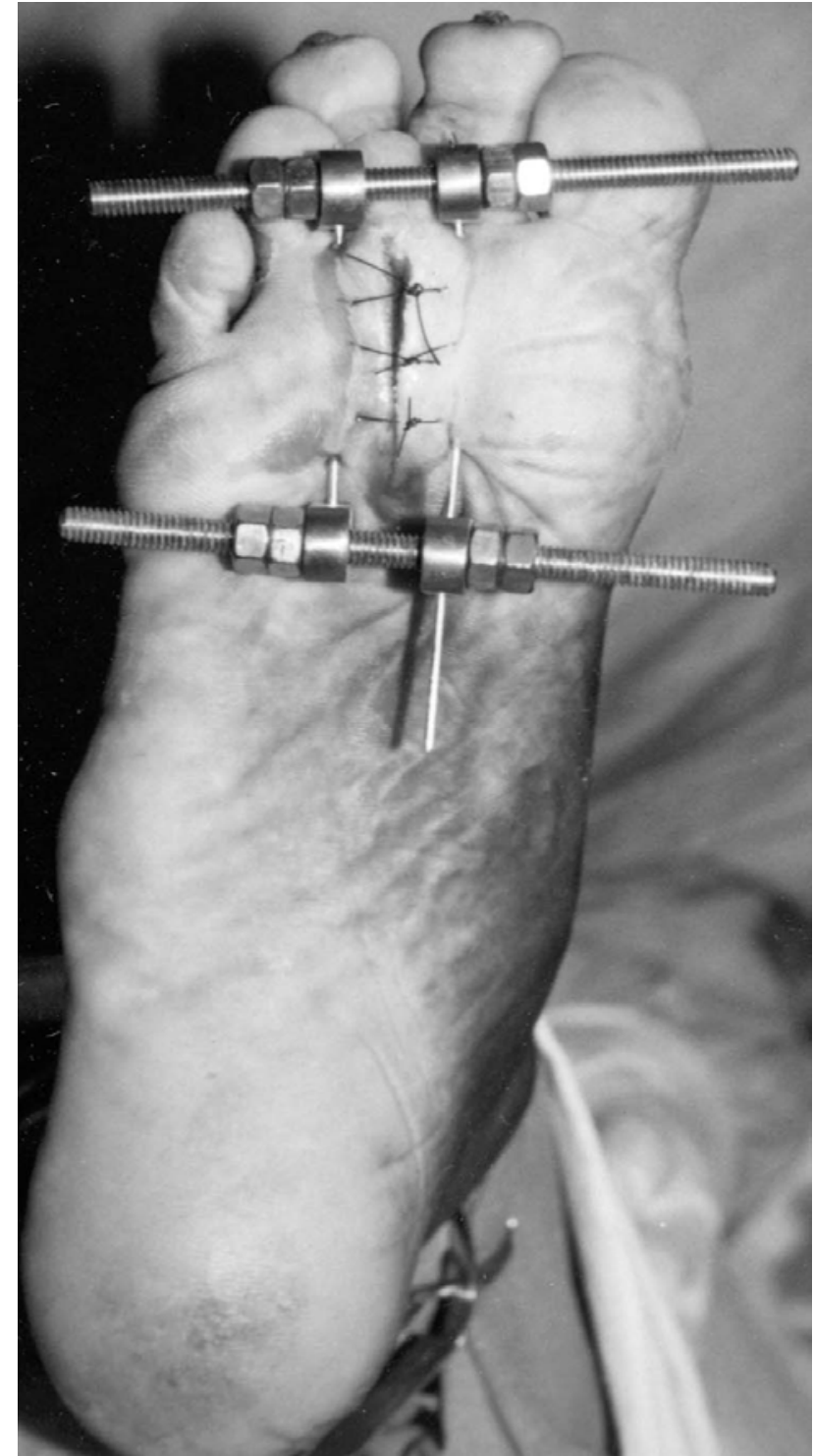

Figure 4 Photograph after the complete approximation was achieved. At this point the edges are sutured together with a strong non-absorbable material.

Although the technique of tissue expansion was first reported by Newman as early as 1957, ${ }^{12}$ it became more popular only after Randovan's 13 description in 1982. In their experimental and clinical study Liang et al introduced the technique of pre-suturing. ${ }^{8}$ 


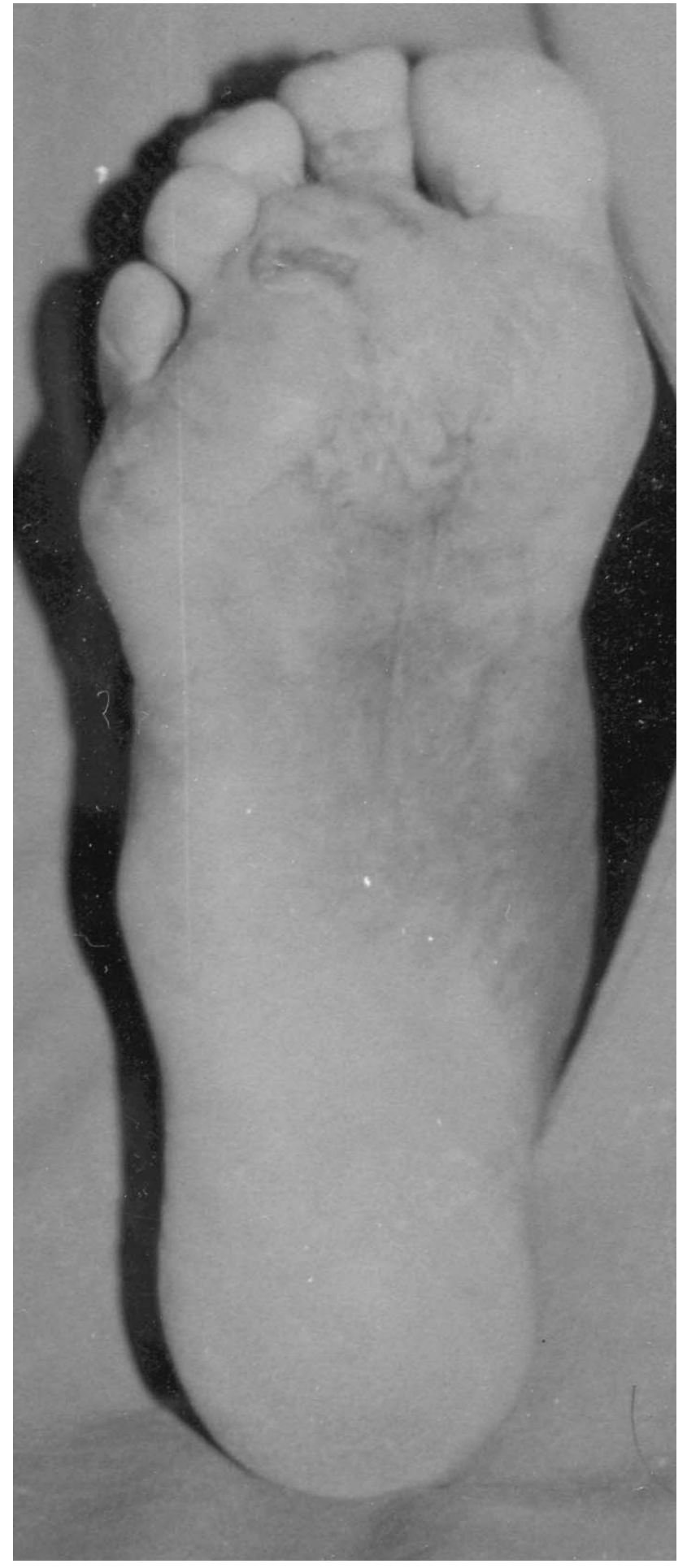

Figure 5 Photograph of the same patient showing the ulcer completely closed.
They described the properties of skin, which contribute to its expansion and can be used to close the skin defect. These include: inherent expansion, mechanical creep and biological creep. Inherent extensibility is defined as the excess skin that allows primary excision and closure. Mechanical creep is a biomechanical property of the skin, which allows it to gradually stretch beyond its limits; this is because of straightening of the normally randomly aligned collagen fibers. Biological creep is the property of skin to increase the tissue by mitotic activity, which has been demonstrated to occur within 24 to 48 hours in response to persistent expansion pressure. ${ }^{14}$

Tissue stretching procedures have been widely used, but its use for the sole of the foot has been infrequently reported. This is probably because of the thick plantar skin. Malaviya has described a technique of closure of simple heel ulcers by skin stretching. ${ }^{15}$ This is an intraoperative technique in which two needles are places on either side of the ulcer and these needles are than approximated by passing sutures around these needles. This technique may be handy in case of small and superficial wounds only. The device designed by the authors has the strength required to approximate the thick plantar skin and has proved effective in closing ulcers as big as $4 \mathrm{~cm}$. The thicker K-wires, which are passed in deeper dermis and are at equal depth throughout the length, provide a strong grip on the skin required for approximating the thick plantar skin. The device provides control on all the four corners of the wound by virtue of the K-wires. Each corner of the wound can be individually and independently subjected to skin stretching. This differential stretching is a very special feature of our device, which is not possible with any device described, even the patented devices marketed. If it is observed that the skin is becoming tight at any one place, the stretching can be stopped only at that place with continuing stretching to other areas. The compressing rods are on either side of the ulcer; contrary to many marketed stretching devices, which have the compressing rod transversing across the wound and allows for easy care of the ulcer. 
The authors device initially uses the mechanical creep to bring about the coverage and probably after two days also induces biological creep accounting for the good results achieved in this report. A patented device-like "sure closure" costs more than 700 dollars per box, but our device will not cost more than a few dollars and can be easily made.

In conclusion, a rather simple solution to overcome otherwise complex situations of skin shortage has been described. We have found that addition of this technique to our armamentarium has effectively increased our options in closure of problematic forefoot plantar ulcers in leprosy.

\section{References}

1. Liwen D, Futian Li, Zaiming W, Juan J, Guocheng Z, Jinhu P, Jugen Z, Yongliang Y. Technique for covering soft tissue defects resulting from plantar ulcers in leprosy: Part IGeneral consideration and summary of results. Indian J Lepr, 1999; 71(3); 285-309.

2. Colen LB, Replogle SL, Mathes SJ. The V-Y flap for reconstruction of forefoot. Plastic Reconstr Surg, 1988; 81(2); 220-228.

3. Lennox WN. Plastic surgery of the anesthetic foot of leprosy. Lepr Rev, 1965; 36(3); 109-117.

4. Giraldo F, De Haro F, Ferrer A. Opposed transverse extended V-Y plantar flaps for reconstruction of neuropathic metatarsal head ulcers. Plastic Reconstr Surg, 2001; 108(4); 1019-1024.

5. Hirshowitz B, Lindenbaum E, Har-Shai Y. A skinstretching device for the harnessing of the viscoelastic properties of skin. Plastic Reconstr Surg, 1993; 92(2); 260269.

6. Stahl S, Har-Shai Y, Hirshowitz B. Closure of wound in the extremity using a skin stretching device. J Hand Surgery, 1996; 21B(4); 534-537.

7. Browne T. Closing a wide wound by using two stout spinal needles and three Allis forceps. Plastic Reconstr Surg, 1998; 101(4); 1159-1161.

8. Liang MD, Briggs P, Heckler FR, Futrell W. PresuturingA new technique for closing large skin defects: Clinical and Experimental study. Plastic Reconstr Surg, 1988; 81(5); 694703.

9. Concannon MJ, Puckett CL. Wound coverage using modified tissue expansion. Plastic Reconstr Surg, 1998; 102(2); 377-384.

10. Abenavoli FM. A simple tissue extensor. Plastic Reconstr Surg, 2002; 109(5); 1763-1764.
11. Melis P, Noorlander ML, van der Horst CMAM, van Noorden CJF. Rapid alignment of collagen fibers in the dermis of undermined and not undermined skin stretching with a skin-stretching device. Plastic Reconstr Surg, 2002; 109(2); 674-680.

12. Neumann CG. The expansion of an area of skin by progressive distention of a subcutaneous balloon. Plastic Reconstr Surg, 1957; 19; 124-128.

13. Radovan C. Breast reconstruction after mastectomy using the temporary expander. Plastic Reconstr Surg, 1982; 69; 185-202.

14. Lew D, Fuseler JW. The effect of stepwise expansion on the mitotic activity and vascularity of subdermal tissue and induced capsule in the rat .J Oral MaxilloFac. Surg, 1991; 49; 848-853.

15. Malaviya GN. Closure of simple heel ulcers by skin stretching. Indian J Lepr, 2005; 77(3): 53-63. 